\title{
NLO properties of a triphenlyguanidine salt: The importance of pseudo- symmetry
}

\author{
Pedro S. Pereira Silvaa,*, Mauro A. Pereira Gonçalves ${ }^{\mathrm{a}, \mathrm{b}}$, Manuela Ramos Silva ${ }^{\mathrm{a}}$, Anna Zawadzka ${ }^{\mathrm{c}}$, \\ Bouchta Sahraoui $^{\mathrm{d}}$, José A. Paixão ${ }^{\mathrm{a}}$ \\ ${ }^{\text {a }}$ CFisUC, Department of Physics, University of Coimbra, P-3004-516, Coimbra, Portugal \\ ${ }^{\mathrm{b}}$ Materials Research and Technology Department, Luxembourg Institute of Science and Technology (LIST), 4 Avenue des Hauts-Fourneaux 5, L-4362, Esch/Alzette, \\ Luxembourg \\ ${ }^{\mathrm{c}}$ Department of Automation and Measurement Systems, Faculty of Physics, Astronomy and Informatics and Centre for Modern Interdisciplinary Technologies, Nicolaus \\ Copernicus University, Grudziadzka 5, 87-100, Torun, Poland \\ d Institut des Sciences et Technologies Moléculaires d'Angers, MOLTECH ANJOU, CNRS UMR 6200, Université d'Angers, 2 Bd Lavoisier, 49045 Angers Cedex, France
}

A R T I C L E I N F O

\section{Keywords:}

Pseudo-symmetry

Nonlinear optics

Maker fringes

SHG

THG

DFT

\begin{abstract}
A B S T R A C T
Using a combined experimental and computational approach, we were able to trace the quasi-vanishing of the second-order nonlinear optical (NLO) properties of a newly synthesized organic salt, bis(triphenylguanidinium) L-malate, to the pseudo-centrosymmetry of its structure. The employed experimental techniques were single crystal X-ray diffraction to determine the structure, "open-aperture" Z-scan technique to measure the nonlinear absorption, Kurtz and Perry powder method and Maker fringes techniques for second- and third-harmonic generation. The molecular hyperpolarizability tensors $(\beta$ and $\gamma)$ were calculated within Density Functional Theory (DFT) and the macroscopic second- and third-order susceptibility tensors were estimated by combining the supermolecule approach with the oriented gas model. The calculations performed with global hybrid GGA and range-separated functionals reproduced the correct order of magnitude of the observed second- and thirdorder susceptibilities.
\end{abstract}

\section{Introduction}

Most of the early nonlinear optical (NLO) materials were based on inorganic crystals like lithium iodate $\left(\mathrm{LiIO}_{3}\right)$, potassium niobate $\left(\mathrm{KNbO}_{3}\right)$ or potassium dihydrogen phosphate (KDP) $\left(\mathrm{KH}_{2} \mathrm{PO}_{4}\right)$. Besides the inorganic NLO materials, strong and durable, organic molecules have also been considered, since they offer a much larger versatility in assembling different crystal structures, enabling the fine tuning of the linear and nonlinear optical properties of the materials [1,2]. Examples of established NLO organic materials are tetrathiafulvalenes, azo-benzenes, phthalocyanines and difluoroboradiazaindacene derivatives [3-7].

Within the large variety of organic materials, the most common chromophores typically contain donor and acceptor groups mediated by a conjugated $\pi$-electron system, through which the charge transfer occurs. However, the dipole-dipole interactions between these dipolar molecules tend to aggregate them in antiparallel arrangements forcing the crystallization in centrosymmetric space groups, with null macroscopic second-order susceptibility. To overcome this handicap Zyss
$[8,9]$ suggested that instead of dipolar (A- $\pi-D)$ molecules, octupolar structures $\left((D-\pi-A)_{3}\right)$ (where $D$ and A refer to the electron-donating and electron-withdrawing groups respectively) should be used. Octupolar molecules have no static permanent dipole moment, thus facilitating noncentrosymmetric crystallization. Another advantage of octupolar molecules is the optimal transfer of the molecular hyperpolarizability components to the macroscopic level $[10,11]$ as exemplified in the TTB (1,3,5-tricyano-2,4,6-tris( $p$-diethylaminostyryl)benzene) crystal [12].

One example of an octupolar chromophore is guanidine, regarded as potentially interesting for second-order NLO applications, as pointed by Zyss et al. with the encapsulation of guanidinium cations between hydrogen L-tartrate anions [13].

In this work we used a guanidine derivative, triphenylguanidine (TPG), that readily forms salts with a large variety of acids [14-21] and a Second Harmonic Generation (SHG) efficiency of 4.7 times that of urea has been observed for the noncentrosymmetric orthorhombic phase of this compound [21]. Within a crystal environment the triphenylguanidinium cation $\left(\mathrm{TPG}^{+}\right.$) loses the perfect 3-fold rotational symmetry [22], but nevertheless the magnitude of the octupolar

\footnotetext{
* Corresponding author.

E-mail address: psidonio@uc.pt (P.S. Pereira Silva).
} 
irreducible component is dominant when compared with that of the vector component [21]. To ensure the crystallization in a noncentrosymmetric space group we also used a co-former with a chiral centre: L-malic acid.

Hence, we present in this paper the crystal structure and the nonlinear optical properties of a novel organic salt, bis(triphenylguanidinium) L-malate. In addition, we compare the experimental NLO results with calculated susceptibilities. The calculations begin with the determination of the nonlinear optical properties of the microscopic units (within DFT) and proceed with a combination of the supermolecule approach with the oriented gas model with two different local-field corrections.

\section{Experimental section}

\subsection{Synthesis and sample preparation}

One mmol of L-malic acid (Aldrich, 97\%) was dissolved in $70 \mathrm{~mL}$ of water and one mmol of $N, N^{\prime}, N^{\prime \prime}$-triphenylguanidine (TCI, 97\%) was dissolved in $30 \mathrm{~mL}$ of ethanol. The ethanolic solution was slowly added to the water solution and the resulting solution was warmed to the boiling point and then left to evaporate under ambient temperature and pressure. Crystals of bis(triphenylguanidinium) L-malate (I) grew from the solution by slow evaporation over a period of a few weeks.

We prepared thin films for the SHG and THG measurements depositing small drops of a $5 \mathrm{mM}$ water solution of (I) on top of glass (Chevallier S.A. glasses) and letting the solution evaporate slowly in a heating plate at $70^{\circ} \mathrm{C}$. The thickness of the films used in the Maker fringes measurements was $800 \mathrm{~nm}$ approximately.

\subsection{Single crystal $X$-ray diffraction}

The diffraction measurement for (I) was carried out with a single crystal on a Bruker APEX II diffractometer using Mo Ka radiation [23]. Data reduction was performed with SMART and SAINT software [23]. Lorenz and polarization corrections were applied. A multi-scan absorption correction was applied using SADABS [24]. The structure was solved by direct methods using SHELXS-97 program [25], and refined on $F^{2} s$ by full-matrix least-squares with SHELXL-97 program [25]. The anisotropic displacement parameters for non-Hydrogen atoms were applied. The Hydrogen atoms were placed at calculated positions and refined with isotropic parameters as riding atoms, with exception of those bonded to Nitrogen or Oxygen atoms that were located in a difference Fourier synthesis at an intermediate stage of the refinement and then allowed to refine as riding atoms.

In this structure, the cations in the asymmetric unit are related by a pseudo-inversion centre. When a crystal structure have non-equivalent atoms imperfectly related by a crystallographic symmetry operator there is usually a strong correlation between those atoms, leading to geometrical distortions or problems with the anisotropic refinement (see chapter 6 of reference [26]). These difficulties can be partially compensated by using restraints and/or constraints. Checking the .lst file we found indeed high correlations between the anisotropic displacement parameters for pairs of atoms from cations related by the pseudo-inversion centre. To obviate this problem, during the structural refinement, the 'rigid bond restraint' DELU was applied to the Carbon atoms of the phenyl groups. The geometries of the pseudo-symmetryrelated cations were restrained with the SAME instruction.

The crystal data and details concerning data collection and structure refinement are given in Table 1.

Because of the weak anomalous scattering at the Mo K $\alpha$ wavelength, the absolute structure could not be determined from the X-ray data, and the enantiomer has been assigned by reference to an unchanging chiral centre in the synthetic procedure.

Atomic coordinates, thermal parameters and bond lengths and angles have been deposited at the Cambridge Crystallographic Data
Table 1

Crystal data and structure refinement of bis(triphenylguanidinium) L-malate.

\begin{tabular}{|c|c|}
\hline Salt & (I) \\
\hline Emp. formula & $\mathrm{C}_{42} \mathrm{H}_{40} \mathrm{~N}_{6} \mathrm{O}_{5}$ \\
\hline Formula weight & 708.80 \\
\hline Temperature (K) & $293(2)$ \\
\hline Wavelength (Å) & 0.71073 \\
\hline Crystal system & Triclinic \\
\hline Space group & $P 1$ \\
\hline$a(\AA)$ & $11.9846(2)$ \\
\hline$b(\AA)$ & $12.7020(2)$ \\
\hline$c(\AA)$ & $13.1431(3)$ \\
\hline$\alpha\left(^{\circ}\right)$ & $96.6840(10)$ \\
\hline$\beta\left(^{\circ}\right)$ & $103.1680(10)$ \\
\hline$\gamma\left(0^{\circ}\right)$ & $91.6080(10)$ \\
\hline Volume $\left(\AA^{3}\right)$ & $1931.82(6)$ \\
\hline$Z$ & 2 \\
\hline Calc. dens. $\left(\mathrm{g} / \mathrm{cm}^{3}\right)$ & 1.219 \\
\hline Abs. coef. $\left(\mathrm{mm}^{-1}\right)$ & 0.082 \\
\hline$F(000)$ & 748 \\
\hline Crystal size (mm) & $0.40 \times 0.26 \times 0.17$ \\
\hline data collec. range & $3.24-28.38^{\circ}$ \\
\hline Index ranges: & $\begin{array}{l}-15<h<16 \\
-16<k<16 \\
-17<l<17\end{array}$ \\
\hline \multicolumn{2}{|l|}{ Reflections: } \\
\hline collected & 31857 \\
\hline unique & 17903 \\
\hline$R$ (int) & 0.0241 \\
\hline Completeness & $99.8 \%$ \\
\hline \multicolumn{2}{|l|}{$\left(\theta=25.00^{\circ}\right)$} \\
\hline Refinement method & Full-matrix least-squares on $F^{2}$ \\
\hline $\begin{array}{l}\text { Data/restraints/ } \\
\text { parameters }\end{array}$ & $17903 / 471 / 955$ \\
\hline$F^{2}$ Goodness-of-fit & 0.969 \\
\hline \multicolumn{2}{|l|}{$R$ indices: } \\
\hline final $[I>2 \sigma(I)]$ & 0.0491 \\
\hline$w R_{2}$ & 0.1187 \\
\hline all data & 0.0988 \\
\hline$w R_{2}$ & 0.1450 \\
\hline Largest diff. peak & 0.297 \\
\hline and hole $\left(e \AA^{-3}\right)$ & -0.200 \\
\hline
\end{tabular}

Centre (CCDC). Any request to the CCDC for this material should quote the full literature citation and the reference number CCDC 1813545. These data can be obtained free of charge via http://www.ccdc.cam.ac. uk/conts/retrieving.html, or from the Cambridge Crystallographic Data Centre, 12 Union Road, Cambridge CB2 1EZ, UK; fax: (+44) 1223-336033; or e-mail: deposit@ccdc.cam.ac.uk.

\subsection{Differential scanning calorimetry (DSC)}

A DSC experiment was performed using a Perkin Elmer Pyris1 calorimeter, with an intracooler cooling unit at $-25{ }^{\circ} \mathrm{C}$ (ethylene glycol-water (1: $1, \mathrm{v} / \mathrm{v})$ cooling mixture). The sample was hermetically sealed in aluminium pan, and as reference, an empty pan was used. A $20 \mathrm{ml} \mathrm{min}^{-1}$ nitrogen purge was employed. Temperature calibration was performed with high-grade standards, namely, biphenyl (CRM LGC 2610, $T_{\text {fus }}=68.93 \pm 0.03{ }^{\circ} \mathrm{C}$ ) and indium (Perkin Elmer, $x=99.99 \%$, $\left.T_{\text {fus }}=156.60{ }^{\circ} \mathrm{C}\right)[27,28]$. Enthalpy calibration was performed with indium $\left(\Delta_{\text {fus }} \mathrm{H}=3286 \pm 13 \mathrm{~J} \mathrm{~mol}^{-1}\right)$ [27]. DSC curves were analyzed with Pyris software version 3.5.

\subsection{UV-vis-NIR absorption spectrum}

The solid-state UV-Vis-NIR absorption spectrum was obtained by diffuse reflectance using a Cary 5000 UV-Vis-NIR spectrophotometer equipped with an integrating sphere. Before the spectrum of a solid (powder) sample was recorded, a baseline was obtained with barium sulphate. 


\subsection{Nonlinear optical techniques}

\subsubsection{Z-scan}

The nonlinear absorption of (I) was studied with "open-aperture" Zscan measurements employing $532 \mathrm{~nm}, 35$ ps laser pulses, following the procedure described elsewhere [29,30]. Concisely, in the Z-scan technique, the transmittance of a sample is measured as it moves along the propagation direction of a focused Gaussian laser beam, consequently experiencing different intensities at each position. By fitting the experimental data according to equations that can be found in the literature $[29,30]$, the nonlinear absorption parameter, $\beta$, the $\operatorname{Im} \chi^{(3)}$ and Im $\gamma$ values can be obtained.

Before the measurements, we performed a calibration of the nonlinear absorption setup using samples of $\mathrm{C60}$ fullerene, which is a wellknown optical limiter. Then, "open-aperture" Z-scan measurements have been performed for several concentrations of (I) and for various incident laser intensities.

\subsubsection{Maker fringes SHG and THG techniques}

The second- and third-order nonlinear optical responses of a thin film of (I) were measured with the SHG and THG Maker fringes techniques in transmission, using a 30 ps diode pumped passively modelocked Nd: $\mathrm{YVO}_{4}$ laser, with a repetition rate of $10 \mathrm{~Hz}$. The intensity and the polarization of the fundamental beam $(1064 \mathrm{~nm})$ exciting the sample were adjusted precisely with a half wave plate and a polarizer. The intensity at the input face of the sample was assumed to have Gaussian temporal and spatial profiles. The laser beam was focused by a lens $(f=250 \mathrm{~mm})$ on the sample, which had been positioned near the focal plane. The film was mounted on a motorized rotational stage allowing the variation of the incident angle with a resolution of $0.5^{\circ}$ around the normal of the incident beam. After passing through a KG3 filter, which cut out the fundamental beam, and interference filters (532 nm for SHG and $355 \mathrm{~nm}$ for THG) to preserve only the SHG/THG signal, the latter was detected with a photomultiplier (PMT), which was connected with a boxcar averager and a computer. Neutral density filters have been always positioned before the PMT to avoid saturation. The so-called Maker fringes [31] were finally obtained by rotating the sample in the range $\pm 60^{\circ}$ to the normal. Maker fringes measurements have been performed for both $\mathrm{s}$ and $\mathrm{p}$ polarizations.

The data analysis of the second order susceptibilities was done by comparison with the SHG intensity of a standard $0.5 \mathrm{~mm}$ thick Y-cut quartz crystal plate. The simplified model of Lee et al. was used [32].

$\frac{\chi^{(2)}}{\chi_{q}^{(2)}}=\frac{2}{\pi} \frac{l_{c, q}}{d} \sqrt{\frac{I^{2 \omega}}{I_{q}^{2 \omega}}}$

where $\chi^{(2)}$ and $\chi_{q}^{(2)}$ denote the second-order nonlinear susceptibilities of the studied material and quartz, respectively, $I^{2 \omega}$ and $I_{q}^{2 \omega}$ are the second harmonic intensities of the material studied and quartz, respectively, $d$ is the film thickness and $l_{c, q}$ is the coherence length of quartz $(20.5 \mu \mathrm{m}$ [33]).

For the analysis of THG data we used the model of Kubodera and Kobayashi [34]. This model compares directly the maximal amplitudes of third harmonic light intensity of the studied material with those of a $1 \mathrm{~mm}$ silica slab as reference. For low absorption, the relation used to determine the magnitude of $\chi^{(3)}$ is given by

$\frac{\chi^{(3)}}{\chi_{S}^{(3)}}=\frac{2}{\pi} \frac{L_{c, S}}{d} \sqrt{\frac{I^{3 \omega}}{I_{S}^{3 \omega}}}$

where $d$ is the sample thickness, $I^{3 \omega}$ and $I_{S}^{3 \omega}$ are the THG intensities of the material studied and silica $\left(I^{3 \omega}=I_{[\text {sample }+ \text { substrate }]}^{3 \omega} I_{[\text {[substrate }]}^{3 \omega}\right)$, respectively, and the coherence length of silica is given by

$L_{c, S}=\frac{\lambda_{\omega}}{6\left(n_{3 \omega}-n_{\omega}\right)}$.

\subsubsection{Kurtz and Perry powder method}

The SHG efficiency of (I) was also evaluated using the Kurtz and Perry powder method [35]. This method requires that the nonlinear optical material is a powder, which is usually easy to obtain, and allows a fast and efficient way of testing the SHG of candidate NLO materials.

The material (I) was mulled to a fine crystalline powder (90-180 $\mu \mathrm{m})$, compacted in a mount and then installed in a sample holder. The sample was then irradiated with an high-power pulsed laser beam with a fundamental wavelength of $1064 \mathrm{~nm}$. The laser pulses were produced by a Nd:YAG laser at low power, $11 \mathrm{~mJ}$ per pulse, with a duration of $10 \mathrm{~ns}$ and a frequency of $10 \mathrm{~Hz}$. In the sample the intensity of the second-harmonic, $I^{2 \omega}$, generated in all directions, is limited only by the sample holder geometry and this SHG light is focused on a concave mirror that collimates the light. The collimated beam is focused on the photomultiplier (PMT) by a bi-convex lens. The photomultiplier voltage and previous filters are optimized to get a good signal-to-noise relation and avoid the saturation of the photomultiplier. This voltage was measured with a digital oscilloscope triggered by the signal itself. The signals were exported to a computer and integrated with a script written with the software Mathematica version 11.0.1. The result of the integral of the signal is proportional to the SHG intensity generated by the tested material, but the real result of SHG efficiency is obtained by comparison with a signal generated by a reference material under the same experimental conditions. For a correct comparison with the urea reference material the measurements were averaged over several laser thermal cycles.

\section{Computational methods}

\subsection{Calculation of microscopic optical properties}

The calculations of the microscopic optical properties were performed with the GAMESS US package [36].

The linear polarizability $(\alpha)$, first hyperpolarizability $(\beta)$ and second hyperpolarizability $(\gamma)$ tensor components, were computed within Density Functional Theory (DFT) with the following functionals:

- Parameter-free generalized gradient approximation (GGA) functional BLYP (Becke exchange [37] + LYP correlation [38]);

- Global hybrid GGA functionals: B3LYP [37, 38, 39] (20\% HartreeFock (HF) exchange) and LYP [38] (a pure correlation functional with $100 \%$ HF exchange);

- Range-separated functional CAM-B3LYP [40] (Coulomb attenuated B3LYP with 19\% HF plus $81 \%$ B88 exchange interaction at shortrange, and $65 \% \mathrm{HF}$ plus 35\% B88 at long-range, with the intermediate region smoothly described through the standard error function with parameter 0.33 ).

All calculations of microscopic optical properties were performed with the $6-311++\mathrm{G}(\mathrm{d}, \mathrm{p})$ basis set and the $\alpha, \beta$ and $\gamma$ tensors were evaluated by finite field (FF) differentiation using an electric field step, $f=0.001$ a.u.

Two microscopic units were considered: two neutral groups each consisting of one L-malate dianion H-bonded to the two closest triphenylguanidinium cations (see Fig. 1), with their relative positions and geometries as in the crystal. The reason for using non-optimized geometries was that the optimized structures of these molecular clusters were quite different from the experimental, since the structure optimizations were performed in vacuum and so do not take in consideration the crystalline environment. These tensors were subsequently used in the oriented gas model to calculate the macroscopic second- and third-order nonlinear optical properties. The microscopic optical properties of the isolated ions were also computed for comparison purposes, using only the CAM-B3LYP functional. 


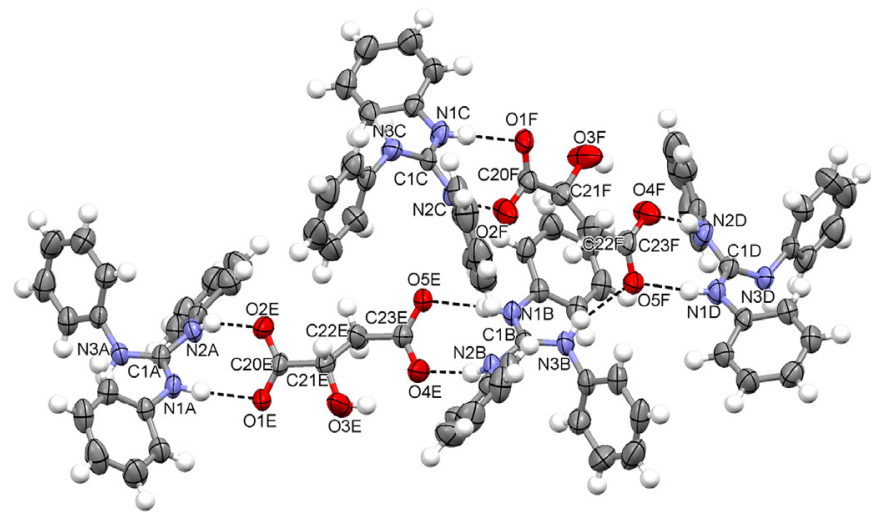

Fig. 1. A plot of the symmetry independent molecular units of bis (triphenylguanidinium) L-malate. Hydrogen bonds are depicted as dashed lines. Displacement ellipsoids are drawn at the $50 \%$ probability level. For clarity, the carbon atoms of the phenyl rings are not labeled.

\subsection{Calculation of macroscopic optical properties}

In our study we used a combination of the oriented gas model with the supermolecule approach to obtain the macroscopic optical properties of (I). In the supermolecule method, the hyperpolarizabilities of a cluster of interacting molecules or ions are calculated as a whole. This approach has the advantage of taking into account the intermolecular interactions within the cluster but it has the disadvantage (for large molecules) of prolonged calculation times, escalating rapidly with the number of units considered. For this reason we chose as the basic units ('supermolecules') the two neutral trimers already mentioned (L-malate dianion $\mathrm{H}$-bonded to the two closest triphenylguanidinium cations). Within this 'supermolecule' the strongest Hydrogen bonds are taken into consideration. The oriented gas model [41] was then used to obtain the macroscopic susceptibilities. Within this model, the crystalline susceptibilities are calculated with a tensor sum of the microscopic hyperpolarizabilities of the molecules ('supermolecules' in this study) that constitute the unit cell, assuming that the intermolecular interactions are much weaker than the intramolecular chemical bonds. For the second-order susceptibility we have the following expression:

$d_{I J K}\left(-\omega ; \omega_{1}, \omega_{2}\right)=\frac{1}{2} \chi_{I J K}^{(2)}\left(-\omega ; \omega_{1}, \omega_{2}\right)$

$=\frac{N}{V} f_{I}(\omega) f_{J}\left(\omega_{1}\right) f_{K}\left(\omega_{2}\right) \frac{1}{N_{g}} \sum_{s} \sum_{i j k} \cos \theta_{I i}^{(s)} \cos \theta_{J j}^{(s)} \cos \theta_{K k}^{(s)}$

$\times \beta_{i j k}^{(s)}\left(-\omega ; \omega_{1}, \omega_{2}\right)$

where $I, J, K$ are the crystal axes, $N_{g}$ is the number of equivalent positions in the unit cell of volume $V$ that has $N$ molecules, $f_{I}(\omega)$ are local field factors for each crystal axis $I$, and the cosine product terms represent the transformation from the molecular reference frame to the crystal frame. The equivalent positions are labeled by the index $s$. The local field factors are basically a correction for the difference between an applied field that would be sensed by the molecule in vacuum and the local field within the material. To calculate the relation between the third-order optical susceptibility $\chi_{I J K L}^{(3)}\left(-\omega ; \omega_{1}, \omega_{2}, \omega_{3}\right)$ and the molecular third-order polarizability, $\gamma_{i j k l}\left(-\omega ; \omega_{1}, \omega_{2}, \omega_{3}\right)$, a generalization of this expression is applied, with one more local field factor for the frequency $\omega_{3}, f_{L}\left(\omega_{3}\right)$. In this study, we calculated the second- and thirdorder macroscopic nonlinear optical properties of (I), using the oriented gas model with the Lorenz-Lorentz (L-L) [42] and the Wortmann and Bishop (W-B) [43] models to calculate the local field factors. The (W-B) model is an extension of Onsager's reaction field model [44] and the details of this method can be found in Ref. [21]. The Wortmann and Bishop local field factors can be regarded as an upgrading over the anisotropic Lorenz-Lorentz [42] spherical cavity expression, generally used to calculate macroscopic NLO properties in crystals, but still falls short from the Rigorous Local Field derived by Munn and coworkers
Table 2

Selected geometry parameters of the four symmetry-independent triphenylguanidinium cations, A, B, C and D $\left(\AA{ }^{\circ}{ }^{\circ}\right)$.

\begin{tabular}{lllll}
\hline & $\mathrm{A}$ & $\mathrm{B}$ & $\mathrm{C}$ & $\mathrm{D}$ \\
\hline C1-N1 & $1.329(6)$ & $1.322(6)$ & $1.328(6)$ & $1.335(6)$ \\
C1-N2 & $1.334(6)$ & $1.334(6)$ & $1.325(6)$ & $1.335(6)$ \\
C1-N3 & $1.334(6)$ & $1.349(6)$ & $1.335(6)$ & $1.336(6)$ \\
N1-C2 & $1.426(6)$ & $1.415(6)$ & $1.407(6)$ & $1.410(6)$ \\
N2-C8 & $1.411(6)$ & $1.417(6)$ & $1.419(6)$ & $1.402(6)$ \\
N3-C14 & $1.406(6)$ & $1.415(6)$ & $1.402(6)$ & $1.431(6)$ \\
N1-C1-N2 & $115.9(4)$ & $116.7(4)$ & $116.8(4)$ & $116.3(5)$ \\
N1-C1-N3 & $121.2(5)$ & $121.1(5)$ & $123.2(5)$ & $123.3(5)$ \\
N2-C1-N3 & $122.9(5)$ & $122.3(5)$ & $120.0(5)$ & $120.3(5)$ \\
C1-N1-C2 & $127.7(4)$ & $127.9(4)$ & $128.6(4)$ & $126.9(4)$ \\
C1-N2-C8 & $127.3(4)$ & $129.2(4)$ & $128.2(4)$ & $128.8(5)$ \\
C1-N3-C14 & $126.9(4)$ & $124.9(4)$ & $127.3(4)$ & $124.4(4)$ \\
C2-N1-C1-N2 & $161.9(4)$ & $-162.4(4)$ & $157.7(5)$ & $-156.8(5)$ \\
C2-N1-C1-N3 & $-17.7(8)$ & $18.5(8)$ & $-22.0(8)$ & $24.4(7)$ \\
C8-N2-C1-N1 & $143.9(5)$ & $-149.5(5)$ & $151.3(5)$ & $-153.9(5)$ \\
C8-N2-C1-N3 & $-36.5(8)$ & $29.6(8)$ & $-29.0(8)$ & $24.9(9)$ \\
C14-N3-C1-N1 & $151.6(5)$ & $-148.1(5)$ & $156.2(5)$ & $-152.8(5)$ \\
C14-N3-C1-N2 & $-28.0(8$ & $32.9(8)$ & $-23.5(8)$ & $28.4(8)$ \\
C1-N1-C2-C3 & $150.1(5)$ & $-149.7(5)$ & $153.2(5)$ & $-152.9(5)$ \\
C1-N2-C8-C13 & $169.2(5)$ & $-164.7(5)$ & $155.6(5)$ & $-153.5(6)$ \\
C1-N3-C14-C15 & $144.6(5)$ & $-146.9(5)$ & $136.5(6)$ & $-138.2(6)$ \\
\hline
\end{tabular}

[45], a more precise method, but computationally much more demanding.

\section{Results and discussion}

\subsection{Crystal structure}

The use of L-malic acid in our synthesis had the objective of forcing the noncentrosymmetry of the crystal structure, to obtain a material with the possibility of SHG, and in fact we succeeded in crystallizing a new noncentrosymmetric salt bis(triphenylguanidinium) L-malate (I). The structure of this salt is triclinic with the noncentrosymmetric space group $P 1$. In this compound, the two carboxyl groups of L-acid are deprotonated giving rise to L-malate dianions. There are four symmetryindependent triphenylguanidinium cations, A, B, C, D, and two symmetry-independent $\mathrm{L}$-malate dianions, $\mathrm{E}$ and $\mathrm{F}$, in the asymmetric unit (Fig. 1). The cations have similar bond lengths and bond angles but are related in pairs (A/B and C/D) by a pseudo-inversion centre so some torsion angles are approximately opposite between these pairs of cations (see Table 2). In the four cations the equivalent angles between the least-squares planes of the guanidinium central fragment and the phenyl rings are identical (see Table 3). The two anions have comparable geometries (see Table 4).

The crystal structure is stabilized by an extensive network of $\mathrm{N}-\mathrm{H} \cdots \mathrm{O}$ hydrogen bonds (see Table 5 ) with each L-malate dianion linked to two triphenylguanidinium cations. There are also intramolecular $\mathrm{O}-\mathrm{H} \cdots \mathrm{O}$ hydrogen bonds in the anions with graph-set descriptor $S_{1}^{1}(6)[46,47]$. The cations and the anions form rings with graph-set $R_{8}^{6}(34)$ [46,47], organizing the structure in layers parallel to the (001) plane (see Fig. 2).

Regarding the relation of the crystal structure with NLO properties of second-order, the presence of the chiral L-malate dianion forced the

Table 3

Angles between the least-squares planes of the guanidinium central fragment and the phenyl rings for the symmetry-independent triphenylguanidinium cations, $\mathrm{A}, \mathrm{B}, \mathrm{C}$ and $\mathrm{D}\left({ }^{\circ}\right.$ ).

\begin{tabular}{lllll}
\hline ring & A & B & C & D \\
\hline C2-C7 & $44.7(1)$ & $45.6(2)$ & $46.3(2)$ & $47.1(2)$ \\
C8-C13 & $47.2(2)$ & $47.7(2)$ & $50.8(2)$ & $50.0(2)$ \\
C14-C19 & $58.2(2)$ & $62.0(2)$ & $61.2(3)$ & $63.8(2)$ \\
\hline
\end{tabular}


Table 4

Selected geometry parameters of the two symmetry-independent L-malate dianions, $\mathrm{E}$ and $\mathrm{F}\left(\AA^{\circ}{ }^{\circ}\right)$.

\begin{tabular}{lll}
\hline & $\mathrm{E}$ & $\mathrm{F}$ \\
\hline $\mathrm{C} 20-\mathrm{O} 1$ & $1.243(6)$ & $1.255(6)$ \\
$\mathrm{C} 20-\mathrm{O} 2$ & $1.260(6)$ & $1.251(6)$ \\
$\mathrm{C} 21-\mathrm{O} 3$ & $1.399(6)$ & $1.405(6)$ \\
$\mathrm{C} 23-\mathrm{O} 4$ & $1.242(6)$ & $1.251(7)$ \\
$\mathrm{C} 23-\mathrm{O} 5$ & $1.260(6)$ & $1.244(6)$ \\
$\mathrm{O} 1-\mathrm{C} 20-\mathrm{O} 2$ & $125.3(5)$ & $125.5(5)$ \\
$\mathrm{O} 4-\mathrm{C} 23-\mathrm{O} 5$ & $123.8(5)$ & $124.7(5)$ \\
$\mathrm{C} 20-\mathrm{C} 21-\mathrm{C} 22-\mathrm{C} 23$ & $-178.8(6)$ & $-178.9(6)$ \\
$\mathrm{O} 1-\mathrm{C} 20-\mathrm{C} 21-\mathrm{O} 3$ & $0.0(7)$ & $0.7(8)$ \\
$\mathrm{O} 1-\mathrm{C} 20-\mathrm{C} 21-\mathrm{C} 22$ & $126.7(5)$ & $127.2(5)$ \\
$\mathrm{O} 3-\mathrm{C} 21-\mathrm{C} 22-\mathrm{C} 23$ & $-54.2(5)$ & $-54.1(5)$ \\
\hline
\end{tabular}

Table 5

Hydrogen-bonding geometry $\left(\AA{ }^{\circ}\right)$ of bis(triphenylguanidinium) L-malate.

\begin{tabular}{|c|c|c|c|c|}
\hline D-H ... A & D-H & $\mathrm{H} \ldots A$ & $\mathrm{D} \ldots \mathrm{A}$ & $D-\mathrm{H} \ldots A$ \\
\hline O3E-H3E ... O4E & 0.82 & 1.93 & $2.636(5)$ & 144.5 \\
\hline O3F-H3F $\cdots$ O4F & 0.82 & 2.02 & $2.648(5)$ & 133.4 \\
\hline N1A-H1A $\ldots$ O1E ${ }^{i}$ & 0.86 & 1.91 & $2.772(5)$ & 172.4 \\
\hline $\mathrm{N} 2 \mathrm{~A}-\mathrm{H} 2 \mathrm{~A} \ldots \mathrm{O} 2 \mathrm{E}^{i}$ & 0.86 & 1.91 & $2.723(6)$ & 158.0 \\
\hline N3A-H3A ... O1F & 0.85 & 2.07 & $2.877(5)$ & 159.0 \\
\hline N3A-H3A ... O3F & 0.85 & 2.33 & $2.885(6)$ & 122.7 \\
\hline N1B-H1B ‥ & 0.85 & 1.96 & $2.797(5)$ & 170.2 \\
\hline N2B-H2B $\cdots \mathrm{O} 4 \mathrm{E}$ & 0.86 & 1.90 & $2.732(5)$ & 163.5 \\
\hline N3B-H3B $\cdots$ O5F $F^{i i}$ & 0.86 & 1.99 & $2.803(5)$ & 156.5 \\
\hline N1C-H1C $\cdots O \mathrm{O}^{i i i}$ & 0.84 & 1.94 & $2.776(5)$ & 171.6 \\
\hline $\mathrm{N} 2 \mathrm{C}-\mathrm{H} 2 \mathrm{C} \cdots \mathrm{O} 2 \mathrm{~F}^{i i i}$ & 0.86 & 1.88 & $2.718(6)$ & 163.3 \\
\hline N3C-H3C $\cdots$ O1E & 0.85 & 2.00 & $2.828(5)$ & 165.4 \\
\hline N3C-H3C $\cdots$ O3E & 0.85 & 2.43 & $2.899(5)$ & 115.6 \\
\hline N1D-H1D ... O5F F $^{i v}$ & 0.87 & 1.93 & $2.785(6)$ & 165.6 \\
\hline $\mathrm{N} 2 \mathrm{D}-\mathrm{H} 2 \mathrm{D} \ldots \mathrm{O} 4 \mathrm{~F}^{i v}$ & 0.85 & 1.87 & $2.703(5)$ & 165.4 \\
\hline N3D-H3D $\ldots$ O5E $\mathrm{E}^{i}$ & 0.86 & 1.93 & $2.756(6)$ & 160.7 \\
\hline
\end{tabular}

Symmetry codes $i: x-1, y, z-1 ; i i: x, y+1, z+1$.

iii: $x, y, z+1 ; i i: x, y+1, z$.

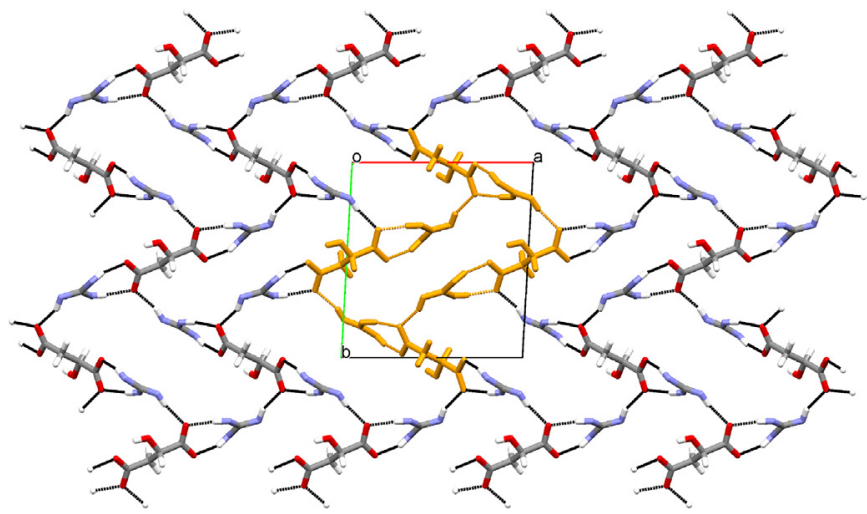

Fig. 2. Packing diagram of (I) viewed down the $c$ axis, showing the layered structure parallel to the plane (001). One $R_{8}^{6}(34)$ ring is highlighted with orange-colored molecules. The hydrogen bonds are depicted as dashed lines and the phenyl rings have been omitted for clarity. (For interpretation of the references to color in this figure legend, the reader is referred to the Web version of this article.)

crystallization of (I) in the noncentrosymmetric space group P1. However, this structure can be viewed as a combination of two sublattices (Fig. 3), a pseudo-centrosymmetric cation sublattice and a truly noncentrosymmetric anion sublattice. As we will see in 4.4.4, the triphenylguanidinium cation has higher hyperpolarizabilities compared with the L-malate dianion so it is expected that most of the second-order NLO response will be canceled due to this pseudo-symmetry. The nonlinear optical effects of third order are not affected by centrosymmetry and in fact the third-order susceptibility tensor $\chi_{I J K L}^{(3)}$ has 81 independent nonzero elements for both classes 1 and $\overline{1}$ of the Triclinic system [48].

\subsection{Differential scanning calorimetry (DSC)}

A typical DSC curve obtained on heating compound (I), performed between 25 and $220^{\circ} \mathrm{C}$, at a scanning rate $\beta=10^{\circ} \mathrm{C} \min 1-$, is shown in Fig. 4. Only one endothermic transition is observed, which is assigned to the fusion process at circa $198{ }^{\circ} \mathrm{C}$ followed by degradation.

\subsection{UV-vis-NIR absorption spectrum}

The solid-state UV-Vis-NIR absorption spectrum of compound (I) was recorded in the range $200-1200 \mathrm{~nm}$ and is presented in Fig. 5.

As we can see, the material is transparent at $1064 \mathrm{~nm}$ and the absorption is very low at $532 \mathrm{~nm}$ and therefore there is no deleterious influence in the SHG. However, there is some absorption at $355 \mathrm{~nm}$, that can have some effect on the THG response. In the calculations of the third-order susceptibility, presented in this study, we did not take this effect into account and we assumed it to be low.

\subsection{Nonlinear optical properties}

\subsubsection{Z-scan}

The nonlinear absorption of compound (I) was investigated with "open-aperture" Z-scan measurements performed for water solutions with concentrations ranging from 0.5 to $2 \mathrm{mM}$. Within experimental accuracy, no two-photon absorption was observed for all concentrations studied.

\subsubsection{Maker fringes $S H G$ and $T H G$ measurements}

The effective second- and third-order nonlinear optical susceptibilities, $\chi^{(2)}$ and $\chi^{(3)}$ respectively, of the salt (I) were measured with the Maker fringes technique.

We did not obtain any SHG signal but this can be explained by two reasons. First of all, the material of the thin films may be not well crystallized and an amorphous material will not show any SHG signal. Another possible explanation for the null result is the pseudo-centrosymmetry of the crystal structure of the salt (I).

For the evaluation of the absolute value of $\chi^{(3)}$, the reference material used was fused silica $\left(2 \times 10^{-22} \mathrm{~m}^{2} / \mathrm{V}^{2}\right)$ [49]. The value of thirdorder NLO susceptibility $\chi^{(3)}$ for (1) at $\lambda=1064 \mathrm{~nm}$ has been estimated to be $(1.77 \pm 0.16) \times 10^{-21} \mathrm{~m}^{2} / \mathrm{V}^{2}$, for the s-s polarization, and $(1.74 \pm 0.07) \times 10^{-21} \mathrm{~m}^{2} / \mathrm{V}^{2}$, for the p-p polarization. These values are one order of magnitude larger than the $\chi^{(3)}$ value of silica, which is the reference material for the THG method. Characteristic experimental curves are shown in Fig. 6 with the signal as a function of the angle of incidence.

\subsubsection{Kurtz and Perry SHG measurement}

Since the null SHG result obtained with the Maker fringes technique may be due to a poor crystallinity of the film sample we tested also a crystalline powder sample of (I) with the Kurtz and Perry powder method. The result was not zero but a very low efficiency of 0.02 times the urea standard. This result has an uncertainty of about $10 \%$ as determined by the variation of the signals between individual measurements.

\subsubsection{Calculated microscopic NLO properties}

The $\alpha_{i j}, \beta_{i j k}$ and $\gamma_{i j k l}$ tensor components were computed with the methodology described in 3.1 (CAMB3LYP/6-311 + + G(d,p)) for the four symmetry-independent triphenylguanidinium cations and the two symmetry-independent L-malate dianions. As we can see from Tables 6 and 7 , on average, the hyperpolarizabilities of the cations are higher than those of the anions. It is also evident that the $\beta_{i j k}$ components of 

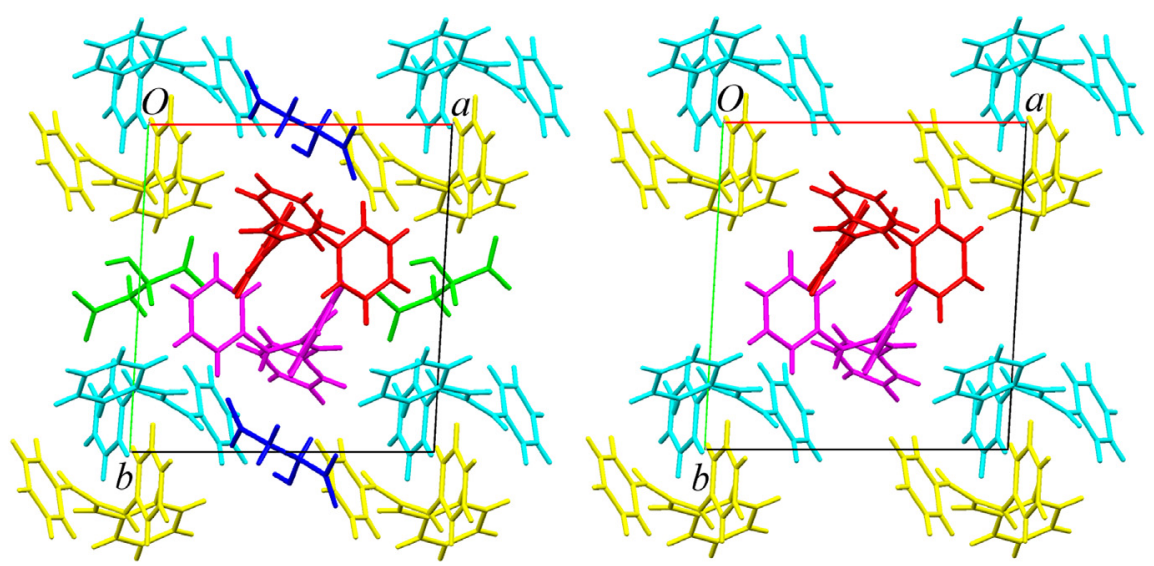

Fig. 3. Left: Packing of (I) viewed down the $c$ axis. Right: The pseudo-centrosymmetric cationic sublattice. The different symmetry-independent ions are identified by colors: $\mathrm{TPG}^{+} \mathrm{A}$ magenta; $\mathrm{TPG}^{+} \mathrm{B}$ red; $\mathrm{TPG}^{+} \mathrm{C}$ cyan; $\mathrm{TPG}^{+} \mathrm{D}$ yellow; $\mathrm{L}-\mathrm{M}^{2-} \mathrm{E}$ green; $\mathrm{L}-\mathrm{M}^{2-} \mathrm{F}$ blue. (For interpretation of the references to color in this figure legend, the reader is referred to the Web version of this article.)

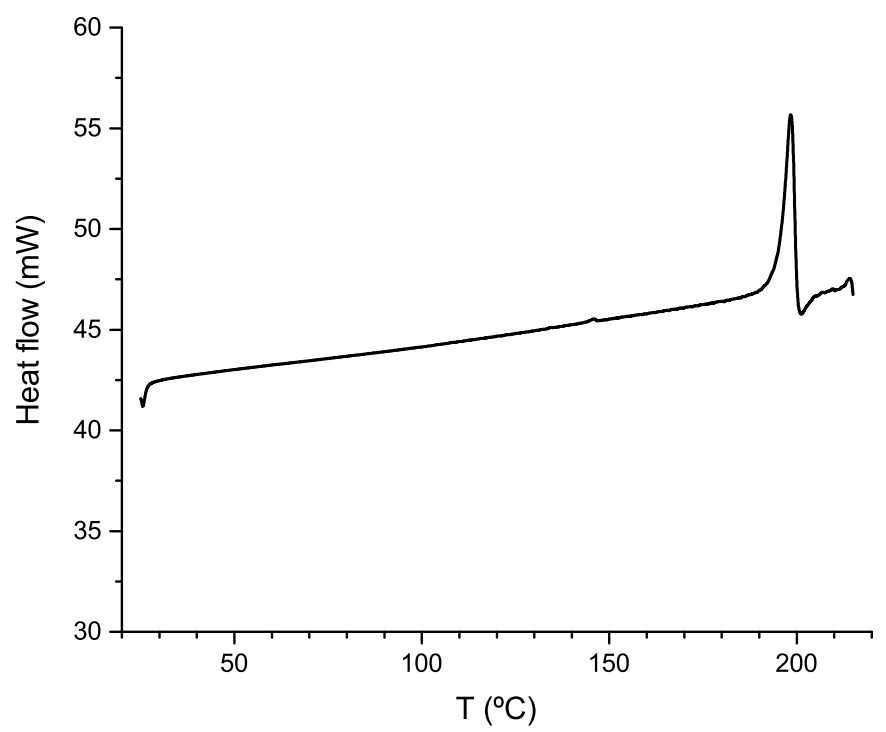

Fig. 4. DSC heating curve of compound (I).

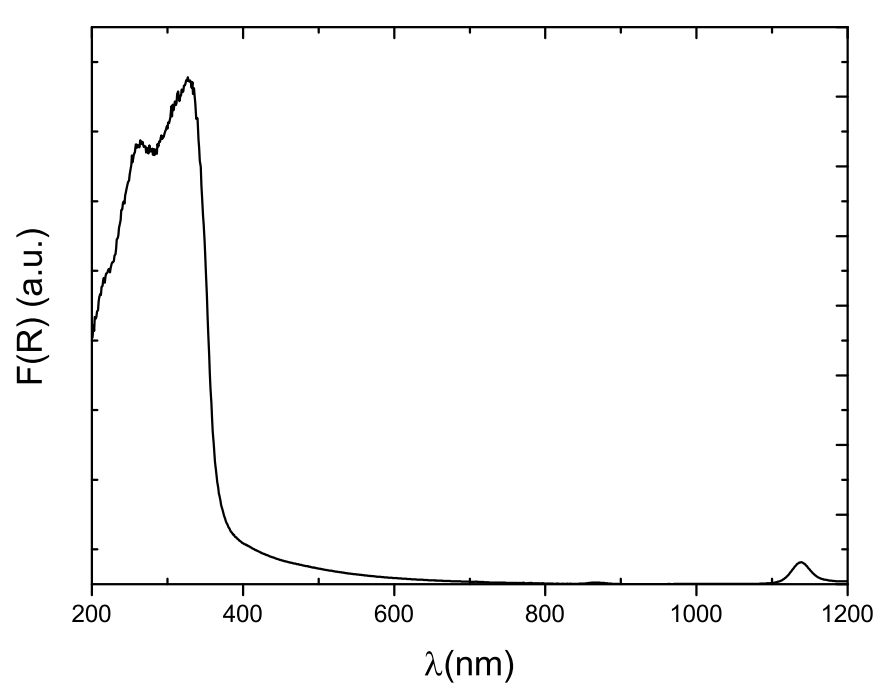

Fig. 5. Solid-state UV-Vis-NIR absorption spectrum of compound (I).

the cations related by the pseudo-inversion centre almost cancel pairwise (A/B, C/D). A similar effect is found for the anion $\beta_{i j k}$ components but to a lesser extent since the $\mathrm{L}$-malate dianions $\mathrm{E}$ and $\mathrm{F}$ are not so perfectly related by the pseudo-symmetry.

To access the deviation from the additivity of the

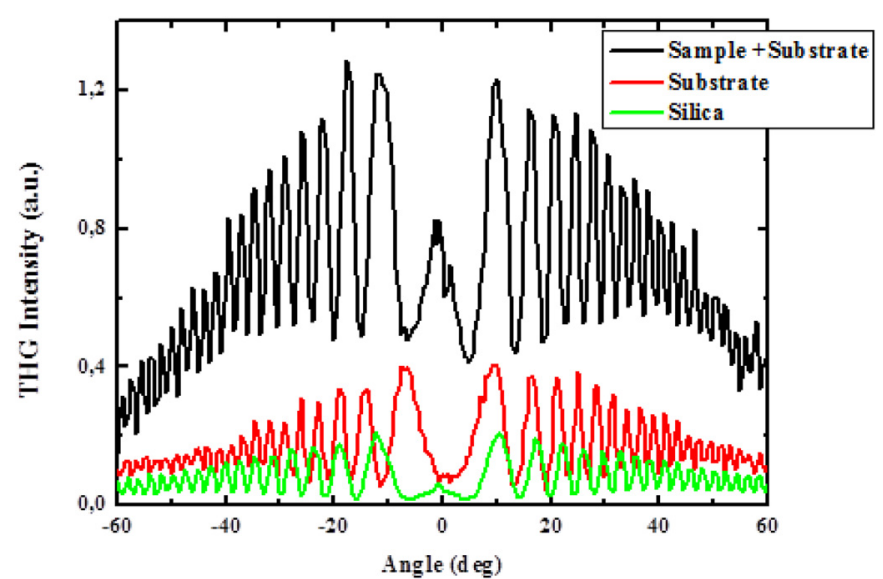

Fig. 6. An example of THG Maker fringes pattern for a film of compound (I), observed by using a Nd-YAG laser of $1064 \mathrm{~nm}$.

Table 6

Calculated values of the 3 strongest components of the first and second hyperpolarizabilities (a.u.) for the four symmetry-independent triphenylguanidinium cations.

\begin{tabular}{lllllll}
\hline & $\beta_{x x z}$ & $\beta_{z z x}$ & $\beta_{z z z}$ & $\gamma_{x x x x}$ & $\gamma_{x x z z}$ & $\gamma_{z z z z}$ \\
\hline A & -410.3 & 217.6 & 461.4 & 48358 & 42777 & 229509 \\
B & 434.3 & -184.3 & -522.9 & 51610 & 44592 & 228241 \\
C & 316.4 & 158.5 & -567.2 & 43800 & 36799 & 228704 \\
D & -311.7 & -136.9 & 559.1 & 43311 & 35670 & 213404 \\
\hline
\end{tabular}

Table 7

Calculated values of the 3 strongest components of the first and second hyperpolarizabilities (a.u.) for the two symmetry-independent s-malate dianions.

\begin{tabular}{lllllll}
\hline & $\beta_{x x z}$ & $\beta_{y y y}$ & $\beta_{z z z}$ & $\gamma_{x x x x}$ & $\gamma_{y y y y}$ & $\gamma_{z z z z}$ \\
\hline $\mathrm{E}$ & -38.3 & -106.7 & -91.8 & 85236 & 44119 & 38073 \\
$\mathrm{~F}$ & 25.5 & 126.7 & 58.1 & 79964 & 44812 & 44048 \\
\hline
\end{tabular}

\section{Table 8}

Calculated values of the 3 strongest components of the first and second hyperpolarizabilities (a.u.) for the two ionic clusters considered compared with sum of the components calculated for the isolated ions.

\begin{tabular}{lllllll}
\hline & $\beta_{x x z}$ & $\beta_{y y y}$ & $\beta_{z z z}$ & $\gamma_{x x x x}$ & $\gamma_{x x z z}$ & $\gamma_{z z z z}$ \\
\hline Cluster 1 & 2.6 & -47.5 & -44.1 & 190646 & 91886 & 419650 \\
Sum (A + E + B) & -14.3 & -108.6 & -153.4 & 185203 & 103946 & 495823 \\
Cluster 2 & 38.8 & 33.5 & 153.4 & 200547 & 36799 & 431734 \\
Sum (C + F + D) & 30.1 & 126.6 & 50.0 & 167075 & 89653 & 486157 \\
\hline
\end{tabular}


Table 9

Theoretical values of the second and third-order susceptibilities estimated with the oriented gas model with L-L and W-B local-field factors, using microscopic optical properties calculated for several levels of theory.

\begin{tabular}{|c|c|c|c|c|c|}
\hline & & $\langle d(\mathbf{I})\rangle$ & $\langle d($ urea $)\rangle$ & \multirow{2}{*}{$\begin{array}{l}\text { SHG(I)/ } \\
\text {-SHG(urea) }\end{array}$} & \multirow{2}{*}{$\frac{\chi^{(3)}}{\left(10^{-21} \mathrm{~m}^{2} / \mathrm{V}^{2}\right)}$} \\
\hline & & $(\mathrm{pm} / \mathrm{V}))$ & $(\mathrm{pm} / \mathrm{V})$ & & \\
\hline BLYP & L-L & 203.7 & 2.01 & 10270 & 667.0 \\
\hline (cluster) & W-B & 96.2 & 1.36 & 5003 & 291.9 \\
\hline B3LYP & L-L & 0.88 & 3.44 & 0.065 & 20.7 \\
\hline (cluster) & W-B & 0.59 & 2.40 & 0.060 & 13.8 \\
\hline LYP & L-L & 0.24 & 3.03 & 0.0063 & 3.94 \\
\hline (cluster) & W-B & 0.17 & 2.27 & 0.0056 & 2.92 \\
\hline CAMB3LYP & L-L & 0.44 & 4.10 & 0.012 & 9.77 \\
\hline (cluster) & W-B & 0.32 & 2.90 & 0.012 & 6.87 \\
\hline CAMB3LYP & L-L & 0.50 & 4.10 & 0.015 & 10.05 \\
\hline (ions) & W-B & 0.35 & 2.90 & 0.015 & 7.79 \\
\hline \multirow{4}{*}{\multicolumn{2}{|c|}{ Experimental }} & \multirow{4}{*}{\multicolumn{2}{|c|}{$\begin{array}{l}\text { (MF s-s) } \\
\sim 0 \\
\text { (MF p- } \\
\text { p) } \sim 0\end{array}$}} & (K-P) 0.02 & (MF s-s) \\
\hline & & & & & $1.77 \pm 0.16$ \\
\hline & & & & & (MF p-p) \\
\hline & & & & & $1.74 \pm 0.07$ \\
\hline
\end{tabular}

hyperpolarizabilities of the ionic units we present in Table 8 the calculated values of the 3 strongest components of the first and second hyperpolarizabilities (a.u.) for the two ionic clusters considered compared with the sum of the components calculated for the isolated ions. The beta values are low so it is difficult to draw any meaningful conclusion about the deviation from the additive behavior, however looking at the second hyperpolarizability components we can see that additivity is a reasonable assumption for this material. Nevertheless, we considered the two clusters as the basic units used in the oriented gas model to calculate the macroscopic properties, so that most of the intermolecular interactions are taken into consideration.

To allow a comparison with the experimental values, the macroscopic NLO properties were computed using the calculated $\beta$ and $\gamma$ tensors.

\subsubsection{Calculated macroscopic $N L O$ properties}

Compound (I) crystalizes in a noncentrosymmetric structure with the triclinic space group $P 1$ and the associated point group 1 . For this point group all the elements of the second-order susceptibility tensor are independent and nonzero, however, by applying the Kleinman symmetry, the number of independent non-zero elements is reduced to 10: $d_{X X X}, d_{Y Y Y}, d_{Z Z Z}, d_{X Y Y}, d_{X Z Z}, d_{Y X X}, d_{Y Z Z}, d_{Z X X}, d_{Z Y Y}$ and $d_{X Y Z}[48,50]$. To calculate these coefficients $d_{I J K}$ of the second-order susceptibility tensor, we have used the $\beta_{i j k}$ values previously determined and the oriented gas model (Eq. (4)) with the Lorenz-Lorentz (L-L) [42] and the Wortmann and Bishop (W-B) [43] local field factors. The local field factors are obtained from the polarizability tensor $\alpha_{i j}$, as explained elsewhere [21]. The angular average of the susceptibility, $\langle d\rangle$, a parameter probed experimentally, is calculated using the expression deduced by Kurtz and Perry [35]:

$\left\langle d^{2 \omega}\right\rangle=\left[\frac{19}{105} \sum_{i}\left(d_{i i i}^{2 \omega}\right)^{2}+\frac{13}{105} \sum_{i \neq j} d_{i i i}^{2 \omega} d_{i j j}^{2 \omega}+\frac{44}{105} \sum_{i \neq j}\left(d_{i i j}^{2 \omega}\right)^{2}\right.$

$\left.+\frac{13}{105} \sum_{i j k, c y c l i c} d_{i i j}^{2 \omega} d_{j k k}^{2 \omega}+\frac{5}{7}\left(d_{i j k}^{2 \omega}\right)^{2}\right]^{1 / 2}$.

More precisely, the experimental result obtained with the Kurtz and Perry method is the ratio between the SHG intensities measured for (I) and for the urea standard. These intensities are proportional to the square of the angular average of the susceptibility, $\langle d\rangle$ (Eq. (5)), so for a meaningful comparison between the calculated and experimental results, calculations for urea with the same methodology as for (I) were also performed.

The third-order susceptibility tensor components were calculated with a generalization of Eq. (4) using the calculated $\gamma_{i j k l}$ values. The average third-order susceptibility for an isotropic material is then given by

$\chi^{(3)}=\frac{1}{5}\left(\chi_{x x x x}^{(3)}+\chi_{y y y y}^{(3)}+\chi_{z z z z}^{(3)}+2 \chi_{x x y y}^{(3)}+2 \chi_{x x z z}^{(3)}+2 \chi_{y y z z}^{(3)}\right)$

The results of all these calculations are presented in Table 9.

We can see from Table 9 that all functionals used were able to describe the correct order of magnitude of the SHG signal with the exception of the parameter-free generalized gradient approximation (GGA) functional BLYP that grossly overestimates the SHG response (it is more advised to rely on the Kurtz-Perry experimental result since the null SHG result obtained with the Maker fringes technique may be due to non-crystallinity of the film sample). It is well-known that rangeseparated functionals and hybrids with a high percentage of HartreeFock (HF) exchange outperform both GGA functionals and global hybrids with low HF exchange [51] in the calculation of NLO properties.

Looking at the $\chi^{(3)}$ we can see a similar trend: a poor performance of the GGA functional and an improvement with a higher percentage of HF exchange. The computational values calculated with the LYP functional are close to the experimental. The $\chi^{(3)}$ results obtained with the L-L factors were generally higher than those calculated with the W-B local field correction but for SHG the difference was negligible for the best performing functionals.

The two CAM-B3LYP calculations (one using two ionic clusters versus the other that considers the sum of the optical properties of the isolated ions) yield results with basically the same quality hinting that, at least for this material, the additivity is a reasonable assumption.

It should be point out that the material is transparent at the SHG wavelength but absorbing at the THG wavelength which can account for part of the discrepancy between the best theoretical values of $\chi^{(3)}$ and the experimental.

\section{Conclusions}

A new organic salt, bis(triphenylguanidinium) L-malate, was synthesized and structurally characterized by single crystal X-ray diffraction. The nonlinear optical properties of this compound were studied with "open-aperture" Z-scan technique, Maker fringes techniques (SHG and THG) and Kurtz and Perry powder method (SHG). The microscopic $\beta$ and $\gamma$ tensor components were calculated for the symmetry-independent ions and for two ionic clusters within DFT, using four functionals from three different families. The macroscopic susceptibilities of second- and third order were estimated using a combination of the supermolecule approach with the oriented gas model with two different local-field corrections. The LYP and CAM-B3LYP functionals were able to reproduce the correct order of magnitude of the experimental second- and third-order susceptibilities. In this study it was possible to correlate the very weak SHG response with the deleterious effect of crystallographic pseudo-symmetry.

\section{Acknowledgements}

The present work was financially supported by FCT Portugal with reference UID/FIS/04564/2016. The authors acknowledge the Laboratory for Advanced Computing at University of Coimbra (http:// www.lca.uc.pt) for providing computing resources that have contributed to the research results reported within this paper.

\section{References}

[1] D.S. Chemla, J. Zyss (Eds.), Nonlinear Optical Properties of Organic Molecules and Crystals, vol. 1, Academic Press, Orlando, FL, 1987.

[2] J. Zyss (Ed.), Molecular Nonlinear Optics. Materials, Physics, and Devices, Academic Press, Boston, MA, 1994.

[3] B. Sahraoui, M. Sylla, J.P. Bourdin, G. Rivoire, J. Zaremba, T.T. Nguyen, M. Sallé, J. 
Mod. Optic. 42 (1995) 2095

[4] I. Guezguez, A. Ayadi, K. Ordon, K. Iliopoulos, D.G. Branzea, A. Migalska-Zalas, M. Makowska-Janusik, A. El-Ghayoury, B. Sahraoui, J. Phys. Chem. C 118 (2014) 7545 .

[5] K. Iliopoulos, I. Guezguez, A.P. Kerasidou, A. El-Ghayoury, D. Branzea, G. Nita, N. Avarvari, H. Belmabrouk, S. Couris, B. Sahraoui, Dyes Pigments 101 (2014) 229.

[6] A. Zawadzka, A. Karakas, P. Płóciennik, J. Szatkowski, Z. Łukasiak, A. Kapceoglu, Y. Ceylan, B. Sahraoui, Dyes Pigments 112 (2015) 116.

[7] B. Kulyk, S. Taboukhat, H. Akdas-Kilig, J.-L. Fillaut, Y. Boughaleb, B. Sahraoui, RSC Adv. 6 (2016) 84854.

[8] J. Zyss, Nonlinear Optic. 1 (1991) 3.

[9] J. Zyss, J. Chem. Phys. 98 (1993) 6583.

[10] J. Zyss, S. Brasselet, V.R. Thalladi, G.R. Desiraju, J. Chem. Phys. 109 (1998) 658.

[11] J. Zyss, I. Ledoux-Rak, H.-C. Weiss, D. Bläser, R. Boese, P.K. Thallapally, V.R. Thalladi, G.R. Desiraju, Chem. Mater. 15 (2003) 3063

[12] V. Le Floc'h, S. Brasselet, J. Zyss, B.R. Cho, S.H. Lee, S.-J. Jeon, M. Cho, K.S. Min, M.P. Suh, Adv. Mater. 17 (2005) 196.

[13] J. Zyss, J. Pécaut, J.P. Levy, R. Masse, Acta Crystallogr. B 49 (1993) 334.

[14] A. Kemme, M. Rutkis, J. Eiduss, Latv. PSR Zinat. Akad. Vestis Kim. Ser. 5 (1988) 595.

[15] P.S. Pereira Silva, J.A. Paixão, M. Ramos Silva, A. Matos Beja, Acta Crystallogr. 62 (2006) 03073.

[16] P.S. Pereira Silva, C. Cardoso, M. Ramos Silva, J.A. Paixão, Acta Crystallogr. 63 (2007) o501.

[17] P.S. Pereira Silva, M. Ramos Silva, J.A. Paixão, A. Matos Beja, Acta Crystallogr. 63 (2007) o2243.

[18] P.S. Pereira Silva, M. Ramos Silva, J.A. Paixão, A. Matos Beja, Acta Crystallogr. 63 (2007) o2524.

[19] L. Dastychova, J. Prihoda, T.J., Collect. Czech Chem. Commun. 72 (2007) 297.

[20] P.S. Pereira Silva, S.R. Domingos, M. Ramos Silva, J.A. Paixão, A. Matos Beja, Acta Crystallogr. 64 (2008) o1082.

[21] P.S. Pereira Silva, C. Cardoso, M.R. Silva, J.A. Paixão, A.M. Beja, M.H. Garcia, N. Lopes, J. Phys. Chem. A 114 (2010) 2607.

[22] C. Cardoso, P.S. Pereira Silva, M.R. Silva, A.M. Beja, J.A. Paixão, F. Nogueira, A.J.F.N. Sobral, J. Mol. Struct. 878 (2008) 169.

[23] Bruker, SMART and SAINT, Bruker AXS Inc., Madison, Wisconsin,USA, 2003.

[24] G.M. Sheldrick, SADABS, University of Göttingen, Germany, 1996.
[25] G.M. Sheldrick, Acta Crystallogr. A 64 (2008) 112.

[26] P. Müller, Crystal Structure Refinement: a Crystallographer's Guide to SHELXL, International Union of Crystallography, Oxford, UK, 2006.

[27] R. Sabbah, X.W. An, J.S. Chickos, M.L.P. Leitão, M.V. Roux, L.A. Torres, Thermochim. Acta 331 (1999) 93.

[28] G. Della Gatta, M.J. Richardson, S.M. Sarge, S. Stolen, Pure Appl. Chem. 78 (2006) 1455.

[29] M. Sheik-Bahae, A.A. Said, T.H. Wei, D.J. Hagan, E.W.V. Stryland, IEEE J. Quant. Electron. 26 (1990) 760.

[30] R. Zaleśny, O. Loboda, K. Iliopoulos, G. Chatzikyriakos, S. Couris, G. Rotas, N. Tagmatarchis, A. Avramopoulos, M.G. Papadopoulos, Phys. Chem. Chem. Phys. 12 (2010) 373.

[31] P.D. Maker, R.W. Terhune, M. Nisenoff, C.M. Savage, Phys. Rev. Lett. 8 (1961) 21

[32] G.J. Lee, S.W. Cha, S.J. Jeon, S.I. Jin, J. Kor. Phys. Soc. 39 (2001) 912.

[33] R.A. Myers, N. Mukherjee, S.R.J. Brueck, Opt. Lett. 16 (1991) 1732.

[34] K. Kubodera, H. Kobayashi, Mol. Cryst. Liq. Cryst. 182 (1990) 103.

[35] S.K. Kurtz, T.T. Perry, J. Appl. Phys. 39 (1968) 3798.

[36] M.W. Schmidt, K.K. Baldridge, J.A. Boatz, S.T. Elbert, M.S. Gordon, J.H. Jensen, S. Koseki, N. Matsunaga, K.A. Nguyen, S. Su, T.L. Windus, M. Dupuis, J.A. Montgomery, J. Comput. Chem. 14 (1993) 1347.

[37] A.D. Becke, Phys. Rev. 38 (1988) 3098.

[38] C. Lee, W. Yang, R.G. Parr, Phys. Rev. B 37 (1988) 785.

[39] A.D. Becke, J. Chem. Phys. 98 (1993) 5648.

[40] T. Yanai, D.P. Tew, N.C. Handy, Chem. Phys. Lett. 393 (2004) 51.

[41] D.S. Chemla, J.L. Oudar, J. Jerphagnon, Phys. Rev. B 12 (1975) 4534.

[42] J. Schwinger, L.L.R. Jr, K.A. Milton, W.Y. Tsai, Classical Electrodynamics, Perseus Books, Cambridge, MA, 1998

[43] R. Wortmann, D.M. Bishop, J. Chem. Phys. 108 (1998) 1001.

[44] L. Onsager, J. Am. Chem. Soc. 58 (1936) 1486.

[45] R.W. Munn, Mol. Phys. 89 (2010) 555.

[46] M.C. Etter, Acc. Chem. Res. 23 (1990) 120

[47] J. Bernstein, R.E. Davis, L. Shimoni, N.L. Chang, Angew Chem. Int. Ed. Engl. 34 (1995) 1555.

[48] R.W. Boyd, Nonlinear Optics, third ed., Academic Press, Burlington, MA, 2008.

[49] U. Gubler, C. Bosshard, Phys. Rev. B 61 (2000) 10702

[50] D.A. Kleinman, Phys. Rev. 126 (1962) 1977.

[51] F. Castet, B. Champagne, J. Chem. Theor. Comput. 8 (2012) 2044. 